\title{
A Review - Automated ECG Analysis using Internet
}

\author{
Apeksha Giri ${ }^{1}$, Baliram Gayal ${ }^{2}$ \\ ${ }^{1,2}$ Indira College of Engineering \& Management, Tal.: Maval, Dist.: Pune, Maharashtra, India
}

\begin{abstract}
This paper discusses about the existing ECG Automated Analysis. A few papers that discuss the automated diagnosis are also studied. Many systems are discussed in these papers about how the patient and Doctor interaction with automated ECG analysis could improve. The techniques are discussed here with overall view of the today's existing systems. How the systems today are helping the doctors and how they could help better is discussed here.
\end{abstract}

Keywords: Automated ECG Analysis, ECG diagnosis, Internet, Review.

\section{Introduction}

Many possible analysis of ECG was carried on in hospital in earlier period. But it is necessary to explore a method to diagnose the patient at home. In various emergency conditions it is necessary to call ambulance as well.

There are many techniques to analyze ECG whether be an analog or the digital. Nowadays Internet based analysis is been used so that doctor's unavailability does not create a problem. Doctor can solve these issues even in his absence in hospital.

\section{Literature Survey}

There are many methods in the past where the internet was the tool used to monitor ECG data. Using the Holter ECG and transmitting the ECG signal on the internet to the remote server and then to the doctor's system was developed [1]. K Y Kong et al. [1] also provide displaying of ECG signals on a various platforms and browsers. Java is mainly used to view the ECG signals.

Further we can also find the automatic detection system for ECG [2] where obtained ECG is processed at the client side into ECG data signals and sent via a web browser to a remote server for further processing that is detection of certain diseases is possible automatically by programming the server.

The processing of ECG [2] is done only using a web browser. That is there is no need to install any software at the client side. The web browser provides the GUI and also allows the server to process the ECG file from the client side. This ECG file may or may not be compressed. This file is sent to the server for processing. That is the file is processed for detection of ECG waveform limits and intervals estimation, late potentials analysis, ischemia monitoring and ischemic changes detection.

Another system [3] in which ECG Signal was checked for whether the patient's condition is normal or critical was used to identify the cluster to which the ECG signal belongs and inform the clinician about the patient's state.
It [3] normally identifies just the cluster to which the ECG signal belongs and sends an alarm message to the clinician and accordingly the clinician will take the respective action.

There is another system [4] where ECG front end hardware acquires, provides filtering of the signal and samples the signal and it is provided to the PDA, where the preliminary diagnosis is provided and in case of very critical situation it calls the emergency ambulance service using the web.

This automated diagnosis works over the various ECG models as references for the software to calculate and display the results. The reliability level depends on the reference model. Initial detection is less reliable as they are based on stored ECG models. But the next identification based on history as well as ECG models gives good reliable results.

The R-peaks are separated or identified by the peak/valley detection algorithm. The parameters preferred are heart rate i.e R-R interval, $\mathrm{P}$ wave duration and amplitude, $\mathrm{P}-\mathrm{R}$ interval for duration, QRS complex for duration and amplitude, Q-T interval for duration. The system [4] diagnosed four types of cardiac disorders, such as sinus tachycardia, sinus bradycardia, ischemia, and myocardial infarction. The patient can use certain services such as printing reports by bluetooth printer, to reporting by e-mail to the doctor or to use the emergency services.

Another system [5] allows remote monitoring of patients by a medical professional. It's main components are, Wireless monitoring device (WMD) which consists of Body area network equipped with various sensors such as $\mathrm{ECG}, \mathrm{SpO} 2$ meter, temperature sensor, and mobility sensor. It also consists of web camera used for monitoring and for interaction between patient and the doctor. All the data obtained from the above network is passed on to the server for processing the data and providing it to the general practitioner or the doctor.

The WMD receives the signals from the sensors provides it to the server. The home monitoring system consisting of WMD and web camera helps to interact with doctor over live video connection. The web server stores all this interaction with the detected signals from the sensors using rich internet applications. 


\section{International Journal of Science and Research (IJSR) \\ ISSN (Online): 2319-7064}

Index Copernicus Value (2013): 6.14 | Impact Factor (2015): 6.391

The web application allows the doctor to remotely login and check each patient's sensor data remotely. Since the data is given in real time, the doctor can interact immediately on this real time data. A reasoning procedure is also embedded in the system. Thus, if critical situation arises and if there is absence of the doctor, the reasoning system should be able to contact the emergency services.

Compressed ECG [6] can also provide ECG diagnosis information compared with decompressed ECG data. The attribute selection is given importance in this system. The system is comprised of the signal acquisition by ECG and sending the compressed ECG packets to the hospital server. The hospital server uses the data mining techniques such as attribute selection and expectation maximization (EM)-based clustering.

The selected attribute set is sent to patient's mobile phone, so the patient's mobile phone can perform analysis. Thus patient's mobile phone does the job of disease estimation using a rule based system.

The difference in various ECG formats [7] representation in the market and no particular standard for them to represent creates a biggest problem while bringing together all these information under one Enterprise information system for management of ECG data.

The ECG formats selected over here are ISO/IEEE11073, Standard Communications Protocol for Computer-assisted electrocardiography, and ECG ontology. Web services, simple object access protocol, extensible markup language, or business process execution language were used to achieve above purpose. The business processes that provide intra and inter country border business extension include Enterprise application integration (EAI), Service oriented architecture (SOA), or Business Process Management (BPM).

Here they have represented communication between personal monitoring ECG devices (1-3 leads) and concentrator devices (such as mobile or PDA). Thus interoperability of devices communicating with each other is a major issue to be solved. A solution they represent here is that they convert the different ECG formats to a central format. In their system Medical device to concentrator device interface ECG format exchange standard was selected to be X73PHD. For the Concentrator to Host System interface the ECG format exchange standard was selected to be SCP-ECG standard. Host system was selected to be ontology based. Host system consists of four main parts such as Java-Servlet, Java-Applet, Database, and Web page.

Likewise studying one more system [8] in which the different ECG formats are mapped to ECG ontology and then encoded in XML and then Internet browser is used to display the ECG.

The cardiac abnormalities that are detected using many systems are in a manner such as one, two, four cardiac abnormalities are detected using ECG models. But a group of cardiac diseases detection has not been possible yet. Even the data that is diagnosed cannot be viewed of one system using the other system. Hence interoperability of the systems regarding ECG formats is necessary. Thus various abnormalities can be detected simultaneously in the ECG output using the author's mentioned system. At most 37 cardiac abnormalities can be detected using the author's system.

Health level 7 standard was selected to be exchange standard. The ontology was derived to achieve a combination of ECG data, HL7 ECG data descriptions, and cardiac diagnosis rules. XML was used to implement the ontology.

The software framework consisted of three parts such as creating XML documents from ECG data, then including the diagnosis in the XML documents, then representing this information in graphical and Text information. Interoperability being the main concern it was checked with numerous ECG files in the Internet Explorer.

\section{Product Survey}

The Mortara RScribe 5 is a PC-based ECG system is used for ECG display \& interpretation by both wireless as well as wired USB system. It can be used on the present PC system and there is no need to buy an additional dedicated PC platform. It can work over network infrastructures as well. Achieving interoperability is done using various industry standard protocols and data formats. HL7 Gateway is also supported for automated data exchange with HIS, EMR systems. Many interpretive statements are also provided to obtain second opinion and help in final diagnosis.

Welch Allyn PC-Based Resting ECG can be provided on single PC or even enterprise wide. ECG measurements that are provided are automatic and can be used to speed clinician review. The clinician can perform checking of ECG from anywhere using the network.

ECG readings are also available using Philips Pagewriter TC30 Electrocardiograph. It has a touch screen which finally increases the speed of accessing ECG data. PDF or XML format is used to represent ECG report. Availability of five minutes ECG patient history allows better creation of ECG reports.

Another application provided by GE CardioSoft Diagnostic System Resting ECG transforms a ordinary PC to a resting ECG device. The ECG report generated by this system is DICOM formatted and can be exported to PACS workstation for faster ECG review. Networking options are also available. Connectivity with other other versions is also facilitated.

PC usage as a resting ECG device is also available using Universal 12-Lead Interpretive ECG machine from QRS Diagnostics. The working software is CardioView ECG Interpretive Software. It also facilitates using of this software on many hospital PCs to enable ECG check in any room. 
All the above mentioned products are costly and most of them are suitable for Hospital environment. Hence these equipment are not preferred for home usage or for monitoring remotely from home.

\section{References}

[1] K Y Kong, CY Ng, K Ong, "Web-Based Monitoring of Real-Time ECG Data", Computers in Cardiology, IEEE Conference, pp. 189-192, 2000.

[2] José García, Ignacio Martínez, Leif Sörnmo, Salvador Olmos, Angel Mur and Pablo Laguna, "Remote Processing Server for ECG-Based Clinical Diagnosis Support", IEEE Transactions on Information Technology, in Biomedicine, Vol. 6, No.4, December 2002, pp. 277-284.

[3] Dong-Her Shih, Hsiu-Sen Chiang, Binshan Lin and Shih-Bin Lin, "An Embedded Mobile ECG Reasoning System for Elderly Patients", IEEE Transactions on Information Technology in Biomedicine, vol. 14, no. 3, May 2010, pp. 854-865.

[4] Claudio De Capua, Antonella Meduri, and Rosario Morello, "A Smart ECG Measurement System Based on Web-Service-Oriented Architecture for Telemedicine Applications", IEEE Transactions on Instrumentation and Measurement, vol. 59, no. 10, october 2010, pp. 2530-2538.

[5] Bingchuan Yuan, John Herbert, "Web-based Real-time Remote Monitoring for Pervasive Healthcare", Pervasive Computing and Communications Workshops (PERCOM Workshops), 2011 IEEE International Conference on, March 2011, pp. 625 - 629.

[6] Fahim Sufi and Ibrahim Khalil, "Diagnosis of Cardiovascular Abnormalities From Compressed ECG: A Data Mining-Based Approach", IEEE Transactions on Information Technology in Biomedicine, vol. 15, no. 1, January 2011, pp. 33-39.

[7] Jesu's Daniel Trigo, Ignacio Mart'inez, A' lvaro Alesanco, Alexander Kollmann, Javier Escayola, Dieter Hayn, G"unter Schreier, and Jos'e Garc'1a, "An Integrated Healthcare Information System for End-toEnd Standardized Exchange and Homogeneous Management of Digital ECG Formats", IEEE Transactions on Information Technology in Biomedicine, vol. 16, no. 4, July 2012, pp. 518-528.

[8] Thidarat Tinnakornsrisuphap and Richard E. Billo, "An Interoperable System for Automated Diagnosis of Cardiac Abnormalities from Electrocardiogram Data", IEEE Journal of Biomedical and Health Informatics, vol. 19, no. 2, March 2015, pp. 493-500.

\section{Author Profile}

Apeksha Giri She is currently pursuing M.E. degree and is a student in E\&TC department, Indira College of Engineering \& Management, Tal.: Maval, Dist.: Pune, Maharashtra, India.

Baliram Gayal He is working as an Assistant Professor, E\&TC Department, in Indira College of Engineering \& Management, Tal.: Maval, Dist.: Pune, Maharashtra, India. 